\title{
Can Weather Influence the Prevalence of Acute-on-chronic Liver Failure?
}

\author{
Matthew McMillin*1,2 \\ ${ }^{1}$ Central Texas Veterans Health Care System, Temple, TX, USA; ${ }^{2}$ Department of Internal Medicine, University of Texas at Austin \\ Dell Medical School, Austin, TX, USA
}

Citation of this article: McMillin M. Can weather influence the prevalence of acute-on-chronic liver failure? J Clin Transl Hepatol 2019;7(4):285-286. doi: 10.14218/JCTH.2019.00058.

Acute-on-chronic liver failure (ACLF) results from an acute insult that occurs in patients with chronic liver disease, ultimately leading to liver failure. The underlying chronic liver dysfunction can be characterized into three categories: chronic liver disease with no cirrhosis; compensated liver cirrhosis; decompensated cirrhosis. The underlying chronic liver diseases are numerous and include alcoholic liver disease, viral hepatitis (types B and C), nonalcoholic fatty liver disease, and other underlying etiologies. ${ }^{1}$ Acute liver injury can arise from viral hepatitis infections (types $A, B$, and $E$ ), acetaminophen toxicity, drug-induced liver injury, toxin exposure, and numerous other hepatic and extrahepatic causes including heat stroke. ${ }^{2}$ Due to the numerous aspects of pathology that can generate both the chronic and acute components of $A C L F$, these patients can have significantly different pathology profiles. This makes ACLF difficult to classify, and this is evident by the different ACLF definitions being used by the Asian Pacific Association for the Study of the Liver, the European Association for the Study of the Liver - Chronic Liver Failure Consortium, and the North American Consortium for the Study of End-Stage Liver Disease with regards to mortality, presence of organ failure, inclusion/exclusion criteria, and other disease characteristics. ${ }^{3,4}$ In addition, these different definitions of ACLF, depending on which definition is used, could potentially influence the outcomes of studies depending upon the metric investigated.

In the current issue, Lin et al. describe that meteorological patterns can have an influence on the prevalence of ACLF, which has previously not been investigated. ${ }^{5}$ In the 3,510 cases that the authors studied from three hospitals in Fuzhou City, China, there was a positive correlation of average humidity and a negative correlation of temperature with the prevalence of $A C L F^{5}$ In addition, the highest prevalence of ACLF was observed in January, followed by July. ${ }^{5}$ Rainfall and change of temperature did not affect the incidence of ACLF. ${ }^{5}$ The authors conclude that low temperature and high humidity contribute to the seasonal pattern of ACLF observed in their patient population. This supports

Abbreviations: ACLF, acute-on-chronic liver failure. Received: 12 December 2019; Accepted: 13 December 2019

*Correspondence to: Matthew McMillin, University of Texas at Austin Dell Medical School, 1601 Trinity Street, Building B, Austin, TX 78701, USA. Tel: +1-512-495-5037, Fax: +1-512-495-5389, E-mail: matthew.mcmillin@austin.utexas.edu that meteorological factors could have an influence on ACLF. In regard to this, it is important to understand whether the direct effects of meteorological changes are inducing ACLF pathology or if these changes are influencing a secondary factor that increases ACLF prevalence.

In regard to the direct influences of meteorological changes on ACLF, hyperthermia has been associated with hepatocyte dysfunction and can lead to a disruption in mitochondria function and reduced oxidative phosphorylation. ${ }^{6}$ In addition, during both non-exertional and exertional hyperthermia, acute liver failure has been observed, though this occurrence is relatively rare. $^{7}$ In regards to lower temperatures, therapeutic hypothermia has been used as a strategy primarily to reduce intracranial hypertension in patients with acute liver failure. However, a recent multicenter clinical study investigating the use of therapeutic hypothermia observed no benefit in reducing brain swelling or increasing the survival rate of acute liver failure patients. ${ }^{8}$ Therefore, as the study by Lin et al. found that lower temperatures were associated with increased prevalence of ACLF, it was likely not due to any direct effect of hyperthermia or hypothermia. 5

While the influence of seasonal meteorological factors on liver diseases is an area that has not been extensively studied, there is some evidence of seasonality towards the viral hepatitis infections of hepatitis A, B, C, and E as they are more prominent in the spring and summer. $^{9}$ In addition, studies outside the liver have shown bacterial infections can be influenced by weather as gastroenteritis hospitalizations in Spain were studied and both hot and cold temperatures increased risk, while precipitation reduced the incidence of hospitalization. ${ }^{10}$ The authors found that certain pathogens were found in different temperatures, such as rotavirus being associated with cold temperatures, and salmonella and other foodborne infections agents associated with high temperatures. ${ }^{10}$ Bacterial infections and bacterial translocation from the gut have been associated with ACLF and bacterial infections are more common in patients with cirrhosis compared to patients without cirrhosis. ${ }^{11}$ Therefore, changes in meteorological factors could increase the rate of viral and bacterial infections in patients with chronic liver disease causing them to progress to ACLF. That being said, more research is needed in this area to identify if this is a causal component of ACLF.

In conclusion, the study by Lin et al. identifies low temperature and high humidity as risk factors for ACLF. ${ }^{5}$ Due to the lack of studies in this area, it is difficult to know the exact mechanisms of these meteorological factors on liver disease 
and function. It is known that changes in temperature and humidity can influence the rate of infection, making this a possible explanation of the seasonality of ACLF. In addition, other seasonal factors, such as Vitamin D and melatonin due to light exposure, could have effects not accounted for entirely by temperature and humidity measures. The seasonality of ACLF, and liver disease in general, is an exciting research area but additional large-scale and well-designed studies are necessary if we are to better characterize the involvement of meteorological measures on ACLF and liver disease.

\section{Acknowledgments}

This study was funded by a VA Career Development award (BX003486) from the United States Department of Veterans Affairs Biomedical Laboratory Research and Development Service to Dr. Matthew McMillin. This material is the result of work supported with resources and the use of facilities at the Central Texas Veterans Health Care System, Temple, Texas. The content is the responsibility of the author(s) alone and does not necessarily reflect the views or policies of the Department of Veterans Affairs or the United States Government.

\section{Conflict of interest}

The author has no conflict of interests related to this publication.

\section{References}

[1] Tsochatzis EA, Bosch J, Burroughs AK. Liver cirrhosis. Lancet 2014;383: 1749-1761. doi: 10.1016/S0140-6736(14)60121-5.

[2] Bernal W, Wendon J. Acute liver failure. N Engl J Med 2013;369:2525-2534. doi: 10.1056/NEJMra1208937.

[3] Sarin SK, Choudhury A, Sharma MK, Maiwall R, Al Mahtab M, Rahman S, et al. Acute-on-chronic liver failure: consensus recommendations of the Asian Pacific association for the study of the liver (APASL): an update. Hepato Int 2019;13:353-390. doi: 10.1007/s12072-019-09946-3.

[4] Hernaez R, Solà E, Moreau R, Ginès P. Acute-on-chronic liver failure: an update. Gut 2017;66:541-553. doi: 10.1136/gutjnl-2016-312670.

[5] Lin S, Han L, Li D, Wang T, Wu Z, Zhang H, et al. The association between meteorological factors and the prevalence of acute-on-chronic liver failure: A population-based study, 2007-2016. J Clin Transl Hepatol 2019;7:341-345. doi: $10.14218 /$ JCTH.2019.00044.

[6] Žūkienè R, Naučienè Z, Šilkūnienè G, Vanagas T, Gulbinas A, Zimkus A, et al. Contribution of mitochondria to injury of hepatocytes and liver tissue by hyperthermia. Medicina (Kaunas) 2017;53:40-49. doi: 10.1016/j.medici. 2017.01.001.

[7] Coenen $S$, Tran $K$, de Haan J, de Man R. Liver transplantation for nonexertional heat stroke-related acute liver failure. BMJ Case Rep 2017 2017: bcr-2017-221029. doi: 10.1136/bcr-2017-221029.

[8] Bernal W, Murphy N, Brown S, Whitehouse T, Bjerring PN, Hauerberg J, et al. A multicentre randomized controlled trial of moderate hypothermia to prevent intracranial hypertension in acute liver failure. J Hepatol 2016;65 273-279. doi: 10.1016/j.jhep.2016.03.003.

[9] Fares A. Seasonality of hepatitis: a review update. J Family Med Prim Care 2015;4:96-100. doi: 10.4103/2249-4863.152263.

[10] Morral-Puigmal C, Martínez-Solanas Ė, Villanueva CM, Basagaña X. Weather and gastrointestinal disease in Spain: A retrospective time series regression study. Environ Int 2018;121:649-657. doi: 10.1016/j.envint.2018.10.003.

[11] Bruns T, Zimmermann HW, Stallmach A. Risk factors and outcome of bacterial infections in cirrhosis. World J Gastroenterol 2014;20:2542-2554. doi: 10.3748/wjg.v20.i10.2542. 